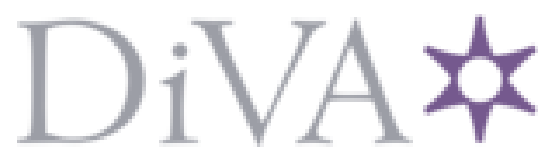

http://www.diva-portal.org

\title{
Postprint
}

This is the accepted version of a paper presented at International Teletraffic Conference 2014 (ITC).

Citation for the original published paper:

Petreska, N., Al-Zubaidy, H., Gross, J. (2014)

Power Minimization for Industrial WirelessNetworks under Statistical Delay Constraints.

In:

http://dx.doi.org/10.1109/ITC.2014.6932953

N.B. When citing this work, cite the original published paper.

Permanent link to this version:

http://urn.kb.se/resolve?urn=urn:nbn:se:kth:diva-158225 


\section{Power Minimization for Industrial Wireless Networks under Statistical Delay Constraints}

\author{
Neda Petreska \\ Fraunhofer Institute for Embedded Systems \\ and Communication Technologies ESK \\ Email: neda.petreska@esk.fraunhofer.de
}

\author{
Hussein Al-Zubaidy James Gross \\ School of Electrical Engineering \\ KTH Royal Institute of Technology \\ Email: hzubaidy@kth.se, james.gross@ee.kth.se
}

\begin{abstract}
Energy efficiency is a very important aspect of modern communication systems. In particular, industrial applications, that deploy wireless machine-to-machine communication and process automation, demand energy-efficient communication in order to prolong battery lifetime and reduce inter-node interference, while maintaining a predefined probabilistic delay bound. In this work, we propose an algorithm that minimizes the transmit power in a WirelessHART network under statistical delay constraints. We achieve this by utilizing a recently developed network calculus approach for wireless networks performance analysis. The evaluation of the algorithm shows that it reaches quasi-minimal power settings within a few iterations.
\end{abstract}

\section{INTRODUCTION}

Energy-efficient wireless networking has received a significant research interest over the last decade. Among other subfields, wireless sensor networking community considers the minimization of energy consumption, which leads to maximizing the lifetime of the network, as a primary design target. Apart from being an academic topic, more recently sensor networks have also become increasingly relevant in practical deployments, which led to the definition of various standards. IEEE 802.15.4 appears to be among the first of such standards emphasizing low-power consumption for wireless networks. Hence, many wireless sensor network architectures are based on IEEE 802.15.4. Wireless sensor networks are nowadays becoming increasingly commercially relevant, especially for industrial applications [1]. Due to this, more specialized standards and protocols have been established (being mostly based on IEEE 802.15.4) such as TSMP [2], WirelessHART [3] and ISA 100 [4]. These standards focus on reliable network operations primarily in the domain of process automation, which requires closed-loop control with relatively relaxed latency requirements in the range of seconds. Nevertheless, the network reliability requirements for such applications, with respect to deadlines in the range of hundreds of milliseconds, needs to be high, usually above $95 \%$. Furthermore, any means to extend the lifetime of the wireless industrial network for such applications is of high interest, as the corresponding plant is usually operated for years (if not for decades), while the control network is supposed to run on batteries. Hence, network maintenance and plant down-time should be at a minimum, requiring an energy efficient operation of the network with respect to the application demands.

The main energy consuming component of a wireless node is usually its wireless transceiver. This suggests that minimizing transmission power would have the desired network energy minimizing effect. However, transmission rate, and hence latency and transmission reliability performance, degrades with the reduction of transmission power. It is therefore necessary to design an algorithm which adapts the transmission power of a wireless node based on the required transmission rate, while still maintaining probabilistic performance bounds.

Wireless networks, on the other side, are generally characterized by randomly varying channel gain due to the effect of fading, which is usually modeled by a random process, e.g., Rayleigh, Nakagami-m, etc. As a result, the channel exhibits time-varying channel capacity. This makes the task of analyzing the performance of wireless networks a challenging one. An approach to address this difficulty is through the use of $(\mathrm{min}, \times)$ stochastic network calculus [5]. This approach allows the representation of random traffic and service processes and provides a methodology to obtain probabilistic performance bounds for wireless networks in terms of the underlying fading channel parameters. The analysis using $(\mathrm{min}, \times)$ network calculus proceeds by transferring the network model into an alternate domain, called the signal-to-noise ratio (SNR) domain, where traffic/service quantities are measured in the amounts of signal-to-noise ratio that is required/available at the receiver side. Hence, this approach permits the analysis of a wireless network where traffic flow is described using a network layer model, while the service offered by the fading channel is described using a physical layer fading channel model. It was shown in [5] that this approach has several advantages over existing approaches for the analysis of wireless systems.

In this work we develop a novel approach and an algorithm for transmission power minimization at a single device in WirelessHART systems. Our objective is to minimize the transmit power while maintaining a target queuing performance of the node, anticipating the target performance to correspond to the quality of service (QoS) demands of an industrial control application. To reach the envisioned goal, we resort to stochastic network calculus (see [5] and references therein), specifically $(\min , \times)$ network calculus, to model and optimize the system performance. The novel application of stochastic network calculus to energy-efficient wireless networks and the developed transmission power minimization algorithm is our main contribution in this paper. This approach exposes fundamental tradeoffs between transmission power efficiency and wireless network performance. More relaxed performance bounds would allow for a more energy-efficient system. It also highlights the effect of channel variability on the achievable probabilistic performance for a given transmission power. In addition, the queuing perspective that we adopt distinguishes this work from much work presented in the domain of physical 
layer design for wireless systems, where the objective is usually to maintain a certain average rate or outage-constrained rate while minimizing the transmission power. When queuing performance is considered in such approaches, the optimization problem becomes much more difficult to track.

The remaining paper is structured as follows. In Section II we present related work. The system model is described in Section III. A brief background for $(\mathrm{min}, \times)$ network calculus is given in Section IV. The power optimization algorithm is presented in Section $\mathrm{V}$ and numerical results are presented in Section VI. Section VII concludes the paper.

\section{RELATED WORK}

The topic of energy minimization under QoS constraints in wireless networks has attracted a lot of interest in the last decade [6]-[12]. Gursoy, Qiao and Velipasalar [6] analyzed the attainable bit energy levels in low power and wideband regimes. They addressed the tradeoff between energy efficiency and spectral efficiency using effective capacity in order to capture QoS behaviour. They also provided energy-bandwidthdelay tradeoff for constant arrival flows in case of no or perfect channel side information (CSI) at the transmitter, while the receiver has only perfect CSI. They concluded that the presence of QoS constraints decreases the spectral efficiency or equivalently increases the energy requirements for fixed spectral efficiency values at low, but non-zero SNR levels. However, direct optimization of the transmit power was not considered. Julian et al. [7] presented a convex optimization framework for resource allocation (power and admission control, delay and throughput optimization) subject to power constraints, data rates and outage probability. The authors of [7] do not, however, consider probabilistic delay bounds. Optimal rate control policy which minimizes the energy consumption for transmitting delay constrained data was addresses by Zafer and Modiano [8]. They obtained a rate adaptation policy that minimizes the transmission energy. This policy is based on a continuous time stochastic control formulation. They further use cumulative curves and obtain an optimal policy with variable deadline constraints, as well as present an energy efficient policy for arbitrary packet arrival. Our work considers transmission power control rather than the rate adaptation used in [8], which is more suitable for the intended application.

Energy-efficient scheduling policy for delay constrained traffic over fading channels was also studied by Lee and Jindal [11]. Near-optimal bit allocation policies are derived for delays bigger than two time slots, while an analytic expression of the optimal scheduler is obtained for a delay equal to two time slots. This work indirectly minimizes the required transmission energy, by controlling the number of transmitted bits per time slot in such way, that the expected energy per time slot is minimized. Fu, Modiano and Tsitsiklis [9] presented an optimal closed-form transmission schedule which maximizes the expected data throughput. This approach was extended to minimization of the energy required to send a fixed amount of data over a fading channel given deadline constraints, when the transmitter has a fixed amount of available energy. The numerical results show a lower energy consumption compared to other threshold policies. However, none of these works maps the obtained policies to practical systems nor focuses on minimizing the transmit power of the nodes.
An energy optimization problem in continuous time for delay constrained data using a calculus approach is formulated by Zafer and Modiano [10]. They considered a queuing system with controllable service rate where the QoS constraints are translated into a minimum departure curve constraint. The authors provide optimal data transmission policies that offer a QoS guarantee over a finite time interval and minimize the expected energy expenditure. They numerically compute the energy cost for any feasible policy. The analysis is done for both deterministic and stochastic arrivals. However, the work is based on deterministic network calculus and stochastic performance bounds over wireless fading channels were not considered. Berry and Gallager [12] tried to regulate both transmission power and buffer delay incurred by the traffic by adapting the transmission rate and power based on the channel state information as well as the buffer occupancy. The authors analyzed the tradeoff between the average delay and the average transmission power required for reliable communication.

Although the mentioned papers focus on energy minimization for delay constrained traffic, none of them considers probabilistic performance bounds for wireless fading channels, while at the same time minimizing the transmit power. In this work we address such performance bounds using the theory of stochastic network calculus and propose an algorithm which determines the minimal transmit power a WirelessHART node should use, in order to satisfy statistical delay constraints and a probability for their violation. To our knowledge, a transmit power optimization algorithm for WirelessHART networks under statistical delay bound constraints does not exist.

\section{SYSTEM MODEL}

Industrial applications deploy WirelessHART for process automation [3]. We present next a brief description of the WirelessHART standard. We also provide description of the underlying wireless channel model and traffic characterization.

\section{A. WirelessHART}

As mentioned earlier, we consider a wireless communication process in a network operating according to the WirelessHART standard for industrial process automation [3]. The generic WirelessHART network topology is presented in Fig. 1. Basically, WirelessHART is a centrally controlled network, where the network manager assigns transmission resources to communication nodes referred to as field devices. It performs these resource allocations based on the application requirements, while trying to maximize the lifetime of the network. Thus, energy-efficient operation of the network depends greatly on the operation of the network manager. The field devices can be sensor and/or actuator nodes and are very often battery-powered. In the following, we focus on a single transmitter and receiver field device pair, which is controlled by the network manager.

WirelessHART employs both direct-sequence spread spectrum (DSSS) and frequency-hopping spread spectrum (FHSS) to mitigate fading and interference. The PHY layer of WirelessHART follows the IEEE 802.15.4-2006 [14] standard for low power devices and networks. The standard uses 16 channels in the $2.4 \mathrm{GHz}$ ISM band and achieves total data rate of up to $250 \mathrm{kbps}$ and a symbol rate of up to $62,5 \mathrm{kBaud} / \mathrm{s}$, while the symbol duration is $16 \mu \mathrm{s}$. At the MAC layer, aside from frequency hopping, WirelessHART employs TDMA, by 


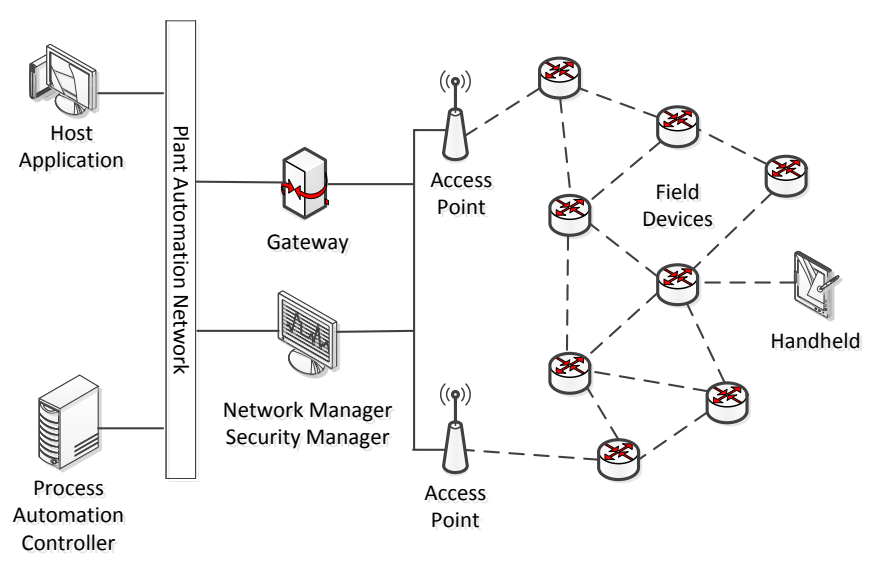

Fig. 1. WirelessHART network architecture [13]

means of which each sender is allocated different time slots for sending data. Each time slot in WirelessHART is $10 \mathrm{~ms}$ long. The TDMA together with the channel hopping is realized by the time synchronized mesh protocol (TSMP) [2] for selforganizing multi-hop networks. In every time slot, a node transmits on a different channel even if it communicates with the same receiver. According to the TSMP time slot format, more than half of each slot is overhead (ACK frame and synchronization preambles). Around $4 \mathrm{~ms}$ (or 250 symbols) remain for payload transmission, which we will consider in the following.

According to the IEEE 802.15.4 standard, a sequence of consecutive and equally sized time slots builds a superframe. A superframe can last from $15 \mathrm{~ms}$ up to several seconds and its format is defined by the network manager. Within every superframe, the network manager allocates specific number of time slots to each device, during which the data is being transmitted.

\section{B. Wireless Radio Channel Model}

As mentioned, we consider the payload communication between a single WirelessHART transmitter $p$ and receiver $q$ via a wireless link. The link is basically modeled as a Rayleigh fading channel and no interference is considered. More precisely, we assume a block-fading process in which the channel gain stays constant within a time slot, but it varies independently between two consecutive slots due to the channel hopping. Let $\left|h_{i}\right|^{2}$ be the instantaneous channel gain in the $i$-th time slot and let $|\bar{h}|^{2}$ be the average channel gain (where the average channel gain results from the path loss, while the instantaneous deviation from this average value is due to fading). Therefore, the received power $P_{\mathrm{rx}}$ and the signal-to-noise ratio $\gamma$ are exponentially distributed [15]:

$$
f(\gamma)=\frac{1}{\bar{\gamma}} e^{-\gamma / \bar{\gamma}}, \gamma \geq 0,
$$

where $f(\gamma)$ is the probability density function of $\gamma$. The average SNR of the channel, $\bar{\gamma}$, is given by:

$$
\bar{\gamma}=\frac{P_{\mathrm{tx}}|\bar{h}|^{2}}{\sigma^{2}},
$$

where $P_{\mathrm{tx}}$ is the transmit power and $\sigma^{2}$ is the noise power. For a known average SNR and average channel state information, the sender can determine the transmit power from Eq. (1). We further assume that the transmitter has knowledge of the average channel gain, as this can be deduced in WirelessHART from the acknowledgments sent back from the receiver immediately after the payload transmission. Finally, we model the instantaneous service $s_{i}$ in time slot $i$, as the maximal instantaneous channel capacity in that time slot, based on the Shannon capacity [16]:

$$
s_{i}=N_{\mathrm{s}} \log _{2}\left(1+\gamma_{i}\right)=\log _{2}\left(1+\gamma_{i}\right)^{N_{\mathrm{s}}},
$$

where $N_{\mathrm{s}}$ is the number of payload symbols that can be transmitted per time slot (in our case $N_{\mathrm{s}}=250$ ) and $\gamma_{i}$ is the instantaneous SNR in the $i$-th time slot.

\section{Traffic Characterization}

The traffic of interest originates from an industrial control application in the field of process automation. Hence, we are interested in a sensor or actuator information flow with low, but constant data rate of $k$ bps. The application has certain quality of service (QoS) requirements which have to be met. These QoS requirements are expressed through the delay $w$ and the probability $\varepsilon$ that this delay is violated. $w$ is given as the maximum number of time slots needed for the application data of the sending node $p$ to reach the application layer of the receiving node $q$. The violation probability represents the maximum percentage of data packets that are not received within the delay $w$, either due to a too low service rate of the wireless channel or due to a corresponding build-up of data in the queue. Typical process automation applications running on top of WirelessHART have delay requirements in the range of several hundreds of milliseconds with a violation probabilitiy in the order of $10^{-3}$ [17].

\section{Problem Formulation and Contribution}

The major goal in this paper is to determine the minimum power used by the transmitter that is required to still serve the information flow with the corresponding quality of service in terms of delay and delay violation probability. By finding the smallest possible average SNR which satisfies such defined QoS parameters, the sender can determine the lowest possible transmit power it should use, according to Eq. (1). Obviously, this will decrease the energy consumption of the node and at the same time decrease the interference with simultaneous neighbouring transmissions.

To account for the fact that a queue builds up at the transmitter from time to time, and the delay and delay violation probability requirements relate to the entire system (transceiver + queue), we resort to stochastic network calculus [18] to model the problem. The theory of network calculus is suitable for describing bounds on the delay for a given service and arrival process characterization. Having the network calculus definition of the delay bound, the major question becomes how to minimize the transmit power (alias the SNR) within the framework, i.e. which method to use to determine the minimum SNR for the required QoS parameters. The main contribution of this work is therefore an algorithm which determines the minimal SNR under the above stated requirements, as a closed-form analytical solution is out of reach given the complexity of the stochastic network calculus framework. The algorithm can be executed by the field devices themselves, as they all have the required input information (they get the 
average channel gain from other field devices and application data related information, like QoS requirements, from the network manager). We will present the binary search-based algorithm after introducing the network calculus model used for our computations in the next section.

\section{Stochastic Network Calculus Approach to WIRELESS NETWORKS ANALYSIS}

The recently developed wireless network calculus based on $(\min , \times)$ dioid algebra is the starting point for the development of our power minimization algorithm for a WirelessHART system. The approach provides probabilistic performance bounds, i.e., delay and backlog, in terms of the average received SNR. The $(\min , \times)$ network calculus relies heavily on the Mellin transform [19] which is defined for any nonnegative random variable $X$ as follows

$$
\mathcal{M}_{X}(s)=E\left[X^{s-1}\right], s \in \mathbb{R},
$$

when the expectation exists.

In the following, we view the network model in the link and network layer as residing in a domain that we call bit domain, while its transformed counterpart in the physical layer in what we call SNR domain. After we introduce the arrival and service process in these two domains, we define the delay bound function by means of Mellin transforms.

\section{A. Arrival and Service Processes in the Bit Domain}

The cumulative arrival to, departure from and service offered by the wireless channel during the time interval $[\tau, t)$ are characterized by the bivariant processes $A(\tau, t), D(\tau, t)$ and $S(\tau, t)$ respectively, which are nonnegative (possibly random) processes and increasing in $t$. Furthermore, we assume causal system where $D(0, t) \leq A(0, t)$.

For a WirelessHART system it is reasonable to assume arrival at a constant rate $k_{\mathrm{ts}}$ bits per time slot. Then we have for any $0 \leq \tau \leq t$

$$
A(\tau, t)=k_{\mathrm{ts}}(t-\tau) .
$$

Similarly, the cumulative departure, i.e., the successfully transmitted bits, is given by

$$
D(\tau, t)=\sum_{i=\tau}^{t-1} d_{i}
$$

where $d_{i}$ denotes the number of successfully transmitted bits during the $i$-th time slot.

The cumulative service (in bits) offered by the underlying wireless fading channel is given by

$$
\begin{aligned}
S(\tau, t) & =\sum_{i=\tau}^{t-1} s_{i}=\sum_{i=\tau}^{t-1} \log \left(1+\bar{\gamma}\left|h_{i}\right|^{2}\right)^{B_{s}} \\
& \triangleq \sum_{i=\tau}^{t-1} \log g\left(\left|h_{i}\right|^{2}\right),
\end{aligned}
$$

where $s_{i}$ is the instantaneous fading channel capacity which is given by Eq. (2) and $B_{s}=N_{\mathrm{s}} / \log 2$. Furthermore, we assume stable operation of the underlying queuing system, where the average arrival rate is smaller than the average service rate of the wireless system, i.e.,

$$
\lim _{t \rightarrow \infty} \frac{A(0, t)}{t} \leq \lim _{t \rightarrow \infty} \frac{S(0, t)}{t} .
$$

Then the delay $W(t)$ experienced at the node is given by

$$
W(t)=\inf _{u \geq 0}\{A(0, t) \leq D(0, t+u)\} .
$$

\section{B. Arrival and Service Processes in the SNR Domain}

In order to apply the $(\min , \times)$ network calculus results, we need to transfer our system description into the SNR domain. Traffic and service processes in the bit domain, i.e., $A, D$ and $S$, are related to their SNR domain counterparts represented by the calligraphic upper case letters $\mathcal{A}, \mathcal{D}$ and $\mathcal{S}$ respectively, through the exponential function, i.e., $\mathcal{A}(\tau, t) \triangleq$ $e^{A(\tau, t)}, \mathcal{D}(\tau, t) \triangleq e^{D(\tau, t)}$ and $\mathcal{S}(\tau, t) \triangleq e^{S(\tau, t)}$.

The cumulative service of a fading channel in the SNRdomain is therefore given by:

$$
\mathcal{S}(\tau, t)=\prod_{i=\tau}^{t-1} g\left(\left|h_{i}\right|^{2}\right) .
$$

Following the definition given with Eq. (5), the delay can be expressed in terms of SNR traffic processes as follows

$$
\mathcal{W}(t)=W(t)=\inf _{u \geq 0}\{\mathcal{A}(0, t) \leq \mathcal{D}(0, t+u)\}
$$

\section{Mellin Transforms for Delay Analysis}

Theorem 1 in [5] expresses the probabilistic delay bound in terms of the Mellin transform of the SNR service and arrival processes. According to that theorem, a probabilistic delay bound $w^{\varepsilon}$ defined for any violation probability $\varepsilon$, i.e., $\operatorname{Pr}\left(W(t)>w^{\varepsilon}\right) \leq \varepsilon$, is the smallest number satisfying

$$
\inf _{s>0}\left\{\mathrm{M}\left(s, t+w^{\varepsilon}, t\right)\right\} \leq \varepsilon,
$$

where

$$
\mathrm{M}(s, \tau, t)=\sum_{u=0}^{\min (\tau, t)} \mathcal{M}_{\mathcal{A}}(1+s, u, t) \cdot \mathcal{M}_{\mathcal{S}}(1-s, u, \tau) .
$$

The minimization over $s$ in Eq. (8) provides the tightest possible bound on the delay violation probability for a given delay bound $w^{\varepsilon}$. Detailed derivation of the bound can be found in [5].

For the deterministic arrival process defined in Eq. (3), we have $\mathcal{A}(\tau, t)=e^{k_{\mathrm{ts}}(t-\tau)}$, which has the Mellin transform

$$
\mathcal{M}_{\mathcal{A}}(s, \tau, t)=\mathrm{E}\left[\mathcal{A}^{s-1}\right]=e^{k_{\mathrm{ts}}(t-\tau)(s-1)} .
$$

Assuming i.i.d fading channel ${ }^{1}$, the Mellin transform for the SNR service process with Eq. (6) is given by

$$
\mathcal{M}_{\mathcal{S}}(s, \tau, t)=\prod_{i=\tau}^{t} \mathcal{M}_{g\left(\left|h_{i}\right|^{2}\right)}=\left(\mathcal{M}_{g\left(\left|h_{i}\right|^{2}\right)(s)}\right)^{t-\tau},
$$

where we used the product property of the Mellin transform. Furthermore, assuming Rayleigh fading, we compute

\footnotetext{
${ }^{1}$ This assumption is valid for block-fading channels which is an acceptable channel model for the WirelessHART system.
} 


$$
\begin{aligned}
P\left(g\left(\left|h_{i}\right|^{2}\right) \leq x\right) & =P\left(\left(1+\bar{\gamma}\left|h_{i}\right|^{2}\right)^{B_{s}} \leq x\right) \\
& =P\left(\left|h_{i}\right|^{2} \leq \frac{x^{1 / B_{s}}-1}{\bar{\gamma}}\right) \\
& =1-e^{-\frac{x^{1 / B_{s}-1}}{\bar{\gamma}}},
\end{aligned}
$$

since for Rayleigh fading, the channel gain $\left|h_{i}\right|^{2}$ is exponentially distributed. The probability distribution function of $g\left(\left|h_{i}\right|^{2}\right)$ is then given by

$$
f_{g\left(\left|h_{i}\right|^{2}\right)}(x)= \begin{cases}\frac{x^{\frac{1}{B_{s}}-1}}{\bar{\gamma} B_{s}} e^{-\frac{x^{1 / B_{s}}-1}{\bar{\gamma}}}, & \text { if } x \geq 1 \\ 0, & \text { otherwise. }\end{cases}
$$

The Mellin transform of $g\left(\left|h_{i}\right|^{2}\right)$ is

$$
\mathcal{M}_{g\left(\left|h_{i}\right|^{2}\right)}(s)=e^{1 / \bar{\gamma}} \cdot \bar{\gamma}^{B_{s}(s-1)} \Gamma\left(B_{s}(s-1)+1, \frac{1}{\bar{\gamma}}\right),
$$

where $\Gamma(s, x)=\int_{x}^{\infty} t^{s-1} e^{-t} d t$ is the incomplete gamma function. It follows that

$$
\mathcal{M}_{\mathcal{S}}(s, \tau, t)=\left[e^{1 / \bar{\gamma}} \cdot \bar{\gamma}^{B_{s}(s-1)} \Gamma\left(B_{s}(s-1)+1,1 / \bar{\gamma}\right)\right]^{t-\tau} .
$$

Substituting Eqs. (10) and (11) in Eq. (9) we have

$$
\begin{aligned}
& \mathrm{M}(s, t+w, t)=\left(e^{1 / \bar{\gamma}} \cdot \bar{\gamma}^{-s B_{s}} \cdot \Gamma\left(1-s B_{s}, \frac{1}{\bar{\gamma}}\right)\right)^{w} \\
& \cdot \sum_{u=0}^{\infty}\left(e^{k_{\mathrm{ts}} s} \cdot e^{1 / \bar{\gamma}} \cdot \bar{\gamma}^{-s B_{s}} \cdot \Gamma\left(1-s B_{s}, \frac{1}{\bar{\gamma}}\right)\right)^{v},
\end{aligned}
$$

where we use the change of variables $v=t-u$ and let $t \rightarrow \infty$. The sum above converges when

$$
e^{k_{\mathrm{ts}} s} \cdot e^{1 / \bar{\gamma}} \cdot \bar{\gamma}^{-s B_{s}} \cdot \Gamma\left(1-s B_{s}, \frac{1}{\bar{\gamma}}\right)<1 .
$$

It follows that

$$
\mathrm{M}(s, \tau, t)=\frac{\left(e^{1 / \bar{\gamma}} \cdot \bar{\gamma}^{-s B_{s}} \cdot \Gamma\left(1-s B_{s}, \frac{1}{\bar{\gamma}}\right)\right)^{w}}{1-e^{k_{\mathrm{ts}} s} \cdot e^{1 / \bar{\gamma}} \cdot \bar{\gamma}^{-s B_{s}} \cdot \Gamma\left(1-s B_{s}, \frac{1}{\bar{\gamma}}\right)} \leq \varepsilon,
$$

for any $s>0$.

The condition given with Eq. (12) is necessary for convergence of the bound in order to obtain bounded violation probability. Otherwise, the delay bound will increase with $t$ and the system becomes unstable. Hence, we refer to this condition as 'stability condition'.

For a WirelessHART pair of nodes $p$ and $q$, communicating over a Rayleigh fading channel, a unidirectional constant data flow that has the QoS requirements $w$ and $\varepsilon$ and based on Eq. (8), we make the following statement: Given a traffic flow of rate $k_{\mathrm{ts}}$, an average SNR $\bar{\gamma}$ and some $s>0$ that satisfy Eq. (12), the probability that the delay exceeds $w$ is upper bounded by $\varepsilon$.

Eq. (13) relates the QoS requirements of the incoming flow to the underlying wireless channel average SNR, and hence to the required transmission power. We propose a transmission power minimization scheme based on Eq. (13) that satisfies the required probabilistic delay bound constraints, i.e.,

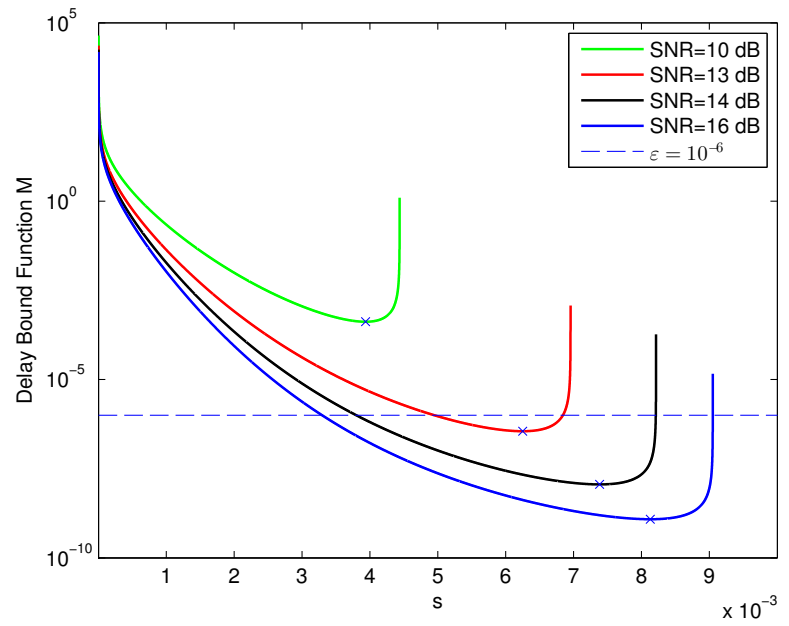

Fig. 2. The delay bound function $\mathrm{M}(s, \bar{\gamma})$ is convex in $s$. Its minimum $s^{*}$ is marked with a cross and shifts to the right as the SNR is increased. The incoming data rate is $5 \mathrm{kbps}$ and the delay constraint is $500 \mathrm{~ms}$. One time slot per superframe is used for transmission and the superframe lasts $100 \mathrm{~ms}$.

Find: $\quad \min \bar{\gamma}$,

subject to: $\quad \inf _{s>0}\{\mathrm{M}(s, \tau, t)\} \leq \varepsilon$

AND $\quad e^{k_{\mathrm{ts}} s} \cdot e^{1 / \bar{\gamma}} \cdot \bar{\gamma}^{-s B_{s}} \cdot \Gamma\left(1-s B_{s}, \frac{1}{\bar{\gamma}}\right)<1$.

Due to the complexity of Eq. (13), an analytical solution for the minimal $\bar{\gamma}$ is not possible. Instead, we propose a binary search algorithm to solve the minimization problem defined above and find the minimum $\bar{\gamma}$ that satisfies the QoS requirements.

\section{Binary Search Algorithm}

In this section we present the main contribution of the paper: An algorithm which finds the optimal average SNR for a given wireless link in an industrial network. Having the optimal SNR, the sending node $p$ can determine the optimal transmit power $P_{\mathrm{tx}}^{*}$ according to Eq. (1). The algorithm is based on binary search in two dimensions: Along $s>0$ and along the average SNR $\bar{\gamma}$. The QoS parameters $w$ and $\varepsilon$, the amount of incoming bits per time slot $k_{\mathrm{ts}}$ and the number of payload symbols $N_{\mathrm{s}}$, that can be transmitted within one time slot, are input parameters of the algorithm. The algorithm uses the convexity in $s$ and the monotonicity in $\bar{\gamma}$ of the delay bound function $M(s, \bar{\gamma})$, where we define $M(s, \bar{\gamma})$ as the right-hand side of Eq. (13). We further define $s^{*}$ as the value of $s$ which minimizes the delay bound function. $s^{*}$ needs to be determined for each new setting, i.e. each time the SNR is either increased or decreased. This behavior is illustrated in Fig. 2.

In more detail, for a fixed $\bar{\gamma}$, the value $s^{*}$ for which $M\left(s^{*}, \bar{\gamma}\right)$ is minimal, is determined by doing binary search along the interval $(0, b)$, where $b$ is the last point for which the stability condition (12) holds. The main idea here is to cut the interval $(0, b)$ into four areas. The initial five chosen points are represented in Fig. 3, where $s_{\mathrm{m}}$ is the middle point of $(0, b)$. Note that we need five points in order to detect an increasing or decreasing trend of $M(s, \bar{\gamma})$. Whenever such trend of $M(s, \bar{\gamma})$ is detected in one of the halves of the interval, this half is discarded. For example, if by comparing the values 


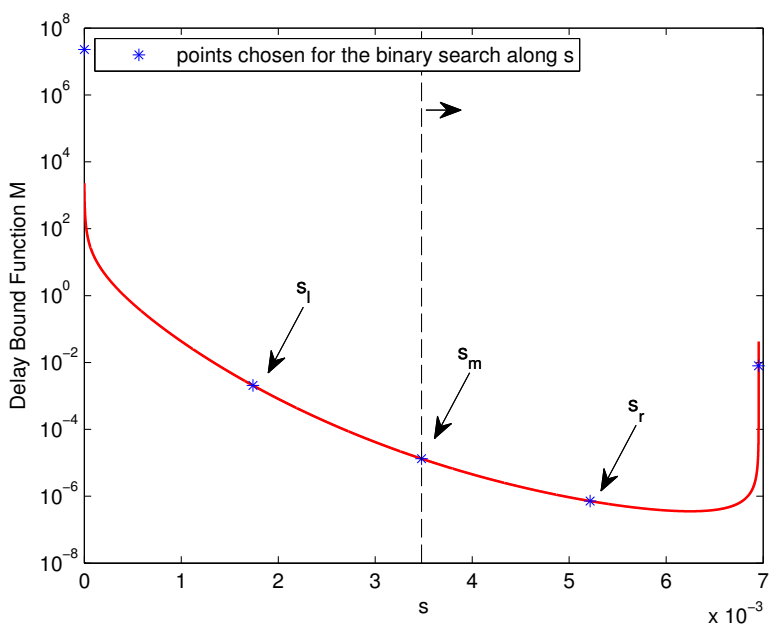

Fig. 3. The chosen points within the interval $(0, b)$ for the binary search along $s . s_{1}$ and $s_{\mathrm{r}}$ mark the middle of the left and right half of the interval, respectively, while $s_{\text {start }}$ and $s_{\text {end }}$ are the points given at the edges of $(0, b)$.

of $\mathrm{M}\left(s_{n}, \bar{\gamma}\right)$, where $s_{n} \in\left\{s_{\text {start }}, s_{1}, s_{\mathrm{m}}, s_{\mathrm{r}}, s_{\text {end }}\right\}$, a decreasing trend is detected (see Fig. 3), then the left half of the interval $(0, b)$ is discarded and the minimum is further searched in the interval $\left(s_{\mathrm{m}}, b\right)$. If an increasing or decreasing trend is detectable from both right and left side of $(0, b)$, then the interval $\left(s_{1}, s_{\mathrm{r}}\right)$ is considered for the next round. These three possible cases during the search phase along $s$ are shown in the pseudo code of the function SEARCH_S $\left(s_{\text {start }}, s_{\text {end }}, \bar{\gamma}, \Delta_{\text {min }}, k_{\mathrm{ts}}, w\right)$ given in Algorithm 2. The function is called recursively until the smallest size of an interval has been reached, defined with the input parameter $\Delta_{\mathrm{min}}$. At this point, the middle point $s_{\mathrm{m}}$ of the last considered interval is returned as $s^{*}$, i.e. as the point $s$ for which $M(s, \bar{\gamma})$ reaches its minimum.

For the search in the second dimension along the average SNR (see Algorithm 1), we start with a low value for $\bar{\gamma}$, which is defined as the lowest possible average SNR that would fulfill Eq. (4), i.e. would result in a channel capacity big enough to transfer $k_{\mathrm{ts}}$ bits per time slot. E.g., one could start with

$$
\bar{\gamma}_{\text {init }}=\left(2^{\frac{k_{\mathrm{ts}}}{N_{\mathrm{s}}}}-1\right)+2
$$

which is derived from the Shannon formula for the channel capacity given with Eq. (2) and then increased by $3 \mathrm{~dB}^{2}$ In case this SNR is not big enough to reach the violation probability, i.e. if $\mathrm{M}\left(s^{*}, \bar{\gamma}\right)>\varepsilon$, then $\bar{\gamma}$ is increased by a factor $f$. In our case, we take $f=2$, i.e. when we increase $\bar{\gamma}$ by 2 , we actually add $3 \mathrm{~dB}$. In case $\mathrm{M}\left(s^{*}, \bar{\gamma}\right)$ lies below $\varepsilon$, i.e. $\mathrm{M}\left(s^{*}, \bar{\gamma}\right)<\varepsilon$, then $\bar{\gamma}$ is decreased for factor $f$ in order to come as close as possible to $\varepsilon$ from below and be able to further minimize the transmit power. Have in mind, that $\bar{\gamma}$ should not be decreased to a value smaller than $\bar{\gamma}_{\text {init }}$ (line 15 in Algorithm 1). The decreasing/increasing factor $f$ is halved whenever a decrease of $\bar{\gamma}$ follows after an increase of $\bar{\gamma}$ and vice versa, in order to avoid that $\bar{\gamma}$ oscillates between two equal values (see lines 5 and 14 in Algorithm 1). In other words, $f \in\left\{2,1,2^{-1}, 2^{-2}, \ldots, f_{\text {stop }}\right\}$. In order to avoid decreasing $f$ infinitely many times, we have introduced the stopping variable $f_{\text {stop }}$ as a further input parameter (line 17 in Algorithm 1).

\footnotetext{
${ }^{2}$ Note that $\bar{\gamma}$ is given as a power ratio in both the algorithm and the theoretical framework.
}

Recall again that the minimum of $\mathrm{M}(s, \bar{\gamma})$ shifts to the right or to the left of the current $s^{*}$, whenever $\bar{\gamma}$ has been increased or decreased, respectively (see Figure 2): After each modification of $\bar{\gamma}$, the function SEARCH_S, which determines the value $s^{*}$ for which $\mathrm{M}\left(s^{*}, \bar{\gamma}\right)$ is minimal, is called again with either $\left(s^{*}, b\right)$ (if $\bar{\gamma}$ was increased) or $\left(0, s^{*}\right)$ (if $\bar{\gamma}$ was decreased), (lines 9 and 25 in Algorithm 1). The algorithm stops modifying $\bar{\gamma}$ as soon as the following condition is met:

$$
\varepsilon-M(s, \bar{\gamma}) \leq \Delta_{\varepsilon} \varepsilon
$$

The so called proximity factor $\Delta_{\varepsilon}$ defines how close we want $\mathrm{M}\left(s^{*}, \bar{\gamma}\right)$ to come to the given violation probability $\varepsilon$ and is also an input parameter of the algorithm. In other words, $\Delta_{\varepsilon}$ can be used as a parameter which sets the desired algorithm precision. The number of iterations needed to reach the near-optimal SNR is increased each time $\bar{\gamma}$ is decreased or increased (represented by the counter $j$ in lines 7 and 23 in Algorithm 1) and is used as an evaluation metric of the algorithm performance in the next section. Depending on $\Delta_{\varepsilon}$ and therefore, on the last value of $f$, the difference between the actual optimal SNR, $\bar{\gamma}_{\text {opt }}$, and the SNR delivered by the algorithm, $\bar{\gamma}^{*}$, is given by

$$
\bar{\gamma}^{*}-\bar{\gamma}_{\text {opt }} \leq f_{\text {last }},
$$

where $f_{\text {last }}$ is the last value of $f$ before the algorithm exits.

The pseudo code of the binary search algorithm is given in Algorithm 1. The function PROVE_STABILITY $\left(s_{\text {start }}, \bar{\gamma}, k_{\mathrm{ts}}\right)$ finds the interval $(0, b)$ for which the stability condition is fulfilled, i.e. finds $b$, such that $\forall s \in(0, b)$ Eq. (12) holds. The function performs a linear search starting from $s_{\text {start }}$ and iterates in small steps $\Delta s$ until it reaches $b+\Delta s$, which violates the stability condition. Note that in order to improve the performance, similarly as in the case of SEARCH_S, PROVE_STABILITY is called with $s_{\text {start }}=0$ or $s_{\text {start }}=s^{*}$ each time $\bar{\gamma}$ was decreased or increased, respectively. Due to simplicity, we omit the pseudo code for this function.

\section{NUMERICAL EVALUATION}

In this section we evaluate the performance of our algorithm for different QoS parameters, as well as incoming data rates, i.e. channel utilizations in a WirelessHART network. We model the system on a time slot basis. We evaluate the performance of the algorithm through the number of iterations needed to compute the optimal SNR for given QoS parameters $(w, \varepsilon)$. This evaluation metric represents the number of times $\bar{\gamma}$ has been increased or decreased until the near-optimal value has been found.

\section{A. Methodology}

In our evaluations we set the superframe duration to 100 $\mathrm{ms}$. The sender $p$ is allocated one time slot per superframe for the data transmission. In the following, we are going to test the algorithm performance and demonstrate its complexity for a wide range of QoS constraints. Please note that the results are showing a rather upper bound on the algorithm behaviour, having in mind the Shannon capacity model we use.

We consider a wireless link between two nodes in a WirelessHART network, on an approximate distance of 20$30 \mathrm{~m}$. The propagation model is represented by the free space path loss model [15], where the path loss exponent is $\alpha=3$, 

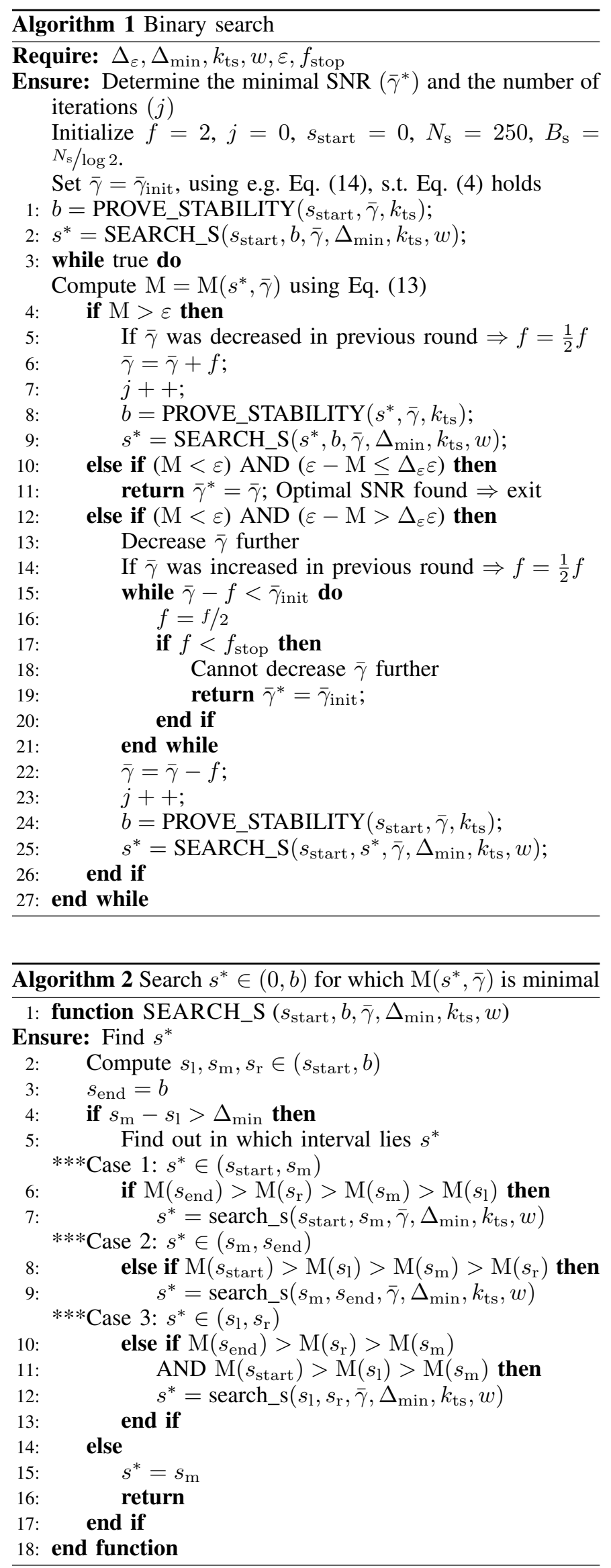

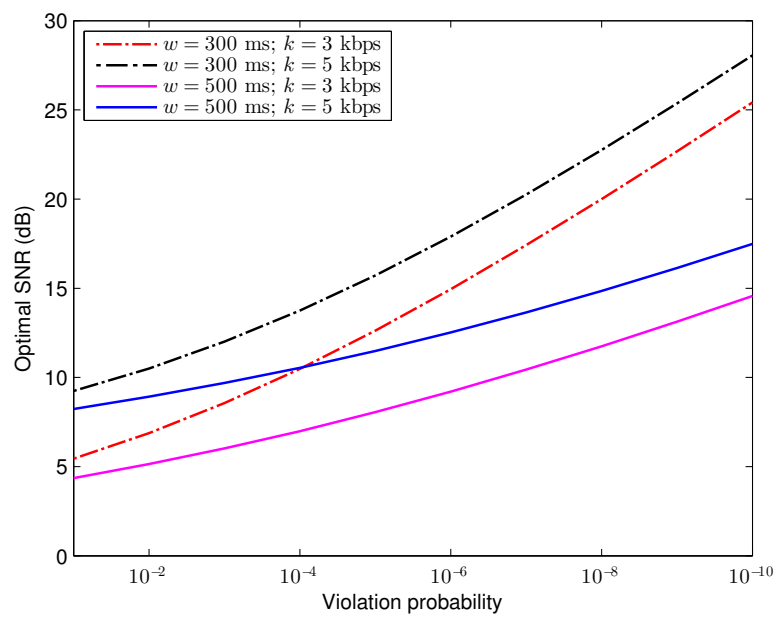

Fig. 4. Optimal SNR for different QoS parameters and incoming data rates.

due to the typical employment of such networks in industrial environments, like for example fabric halls. We set $\Delta_{\min }=$ $10^{-10}$ for the smallest search interval $\left(s_{\text {start }}, b\right), \Delta_{\varepsilon}=0.01$ as the proximity factor in Eq. (15) and use a step of $\Delta s=10^{-6}$ for the linear search of $b$ in PROVE_STABILITY. We stop decreasing the factor $f$ as soon as it gets smaller than $f_{\text {stop }}=$ $2^{-20}$. It is important to mention that we do not increase $f$ to a value bigger than 2 , even if $\bar{\gamma}$ has been increased in two successive iteration rounds. This results into an increased number of iterations for stricter QoS parameters. However, when considering WirelessHART-based control applications, it seems as a reasonable assumption the factor $f$ to have a maximal step of 2. Hence, in case the target application imposes stricter QoS requirements and bigger values of $\bar{\gamma}$ are in general expected, one can double $f$ each time $\bar{\gamma}$ is successively increased, in order to potentially lower the total number of iteration rounds. Therefore, the factor $f$ can be adjusted according to the target control application.

\section{B. Results}

Let us now take a look at the performance of the algorithm. Fig. 4 represents the optimal SNR in dB computed by the algorithm for different target delay bound violation probabilities, where $k \in\{3,5\} \mathrm{kbps}$ and the delay bound $w \in\{300,500\} \mathrm{ms}$. As expected, the optimal average SNR is higher for smaller delays and violation probabilities, as well as for bigger data rates. Hence, for $w=300 \mathrm{~ms}$ and $\varepsilon=10^{-8}$ as well as incoming data rate of $5 \mathrm{kbps}$, the minimal average SNR is around $22 \mathrm{~dB}$. Note that we represent here the SNR and not the transmit power. As stated before, after computing $\bar{\gamma}^{*}$, the sending node can set its transmit power $P_{\mathrm{tx}}^{*}$ according to Eq. (1).

Fig. 5 shows the minimal $\bar{\gamma}$ vs. the target delay for different violation probabilities and data rates. Especially in the area of small delay bounds we notice that the violation probability influences the choice of optimal SNR more than the data rate. Both $2 \mathrm{kbps}$ and $5 \mathrm{kbps}$ curves converge to the minimal possible average SNR, which is defined with $\bar{\gamma}_{\text {init }}$, as given in Eq. (14). The value of the resulting SNR should not be lower than $\bar{\gamma}_{\text {init }}$ for a given incoming data rate $k$, as otherwise, the channel capacity will be smaller than $k$ and the channel will be overutilized. As expected, the minimal allowed SNR, $\bar{\gamma}_{\text {init }}$, is reached sooner for lower data rate flows. 


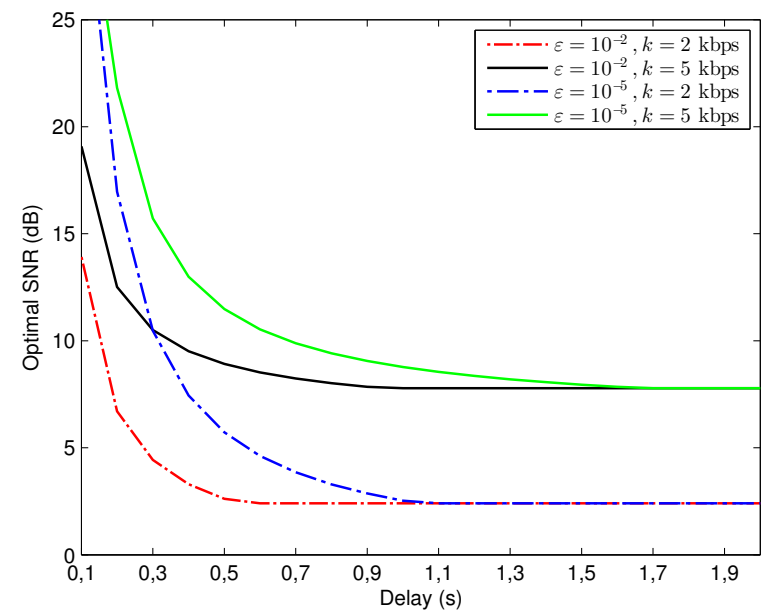

Fig. 5. Optimal SNR vs. delay constraint for different data rates and violation probabilities.

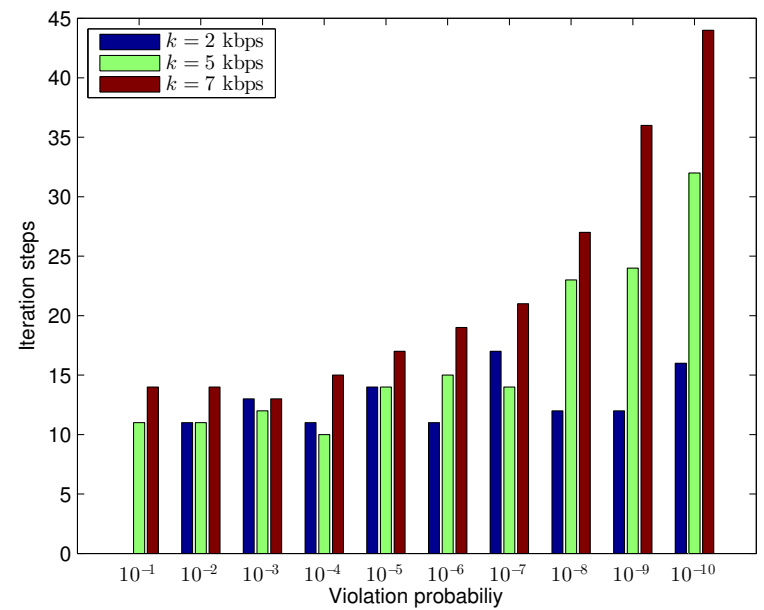

Fig. 6. Algorithm performance for various violation probabilities and data rates, where $w=500 \mathrm{~ms}$. The increasing trend is especially seen for bigger data rates, while the number of iterations for smaller data rate traffic fluctuates.

Let us now look into the number of iterations that the algorithm needs in order to compute $\bar{\gamma}^{*}$. Fig. 6 shows the number of iterations for different target violation probabilities and different data rates, where $w=500 \mathrm{~ms}$. As expected, the number of iterations grows as the target violation probability decreases. We also observe that in case of higher data rates, i.e. higher $\bar{\gamma}^{*}$, the algorithm needs more iterations until the stopping criteria (15) is fulfilled (recall that the maximal increase step is $3 \mathrm{~dB}$ ). Hence, the increase in the number of iterations is more obvious for higher data rates, in comparison to lower ones, where it fluctuates. Note further, that for $\varepsilon=10^{-1}$ and $k=2 \mathrm{kbps}$, the optimal SNR is already reached with $\bar{\gamma}_{\text {init }}$ and therefore, no iterations are needed.

Fig. 7 shows the number of iterations dependent on the delay bound $w$, again for different data rates, in case when $\varepsilon=10^{-4}$. For $w=0,1 \mathrm{~s}$ the number of iterations is very big and exceeds 50 . The algorithm needs less iterations as the delay bound becomes less strict. Notice again, that the number of iterations can fluctuate, especially when the resulting $\bar{\gamma}^{*}$ is not very high, as in the case of lower data rates. The number of iterations depends on the proximity factor $\Delta_{\varepsilon}$ and the stopping

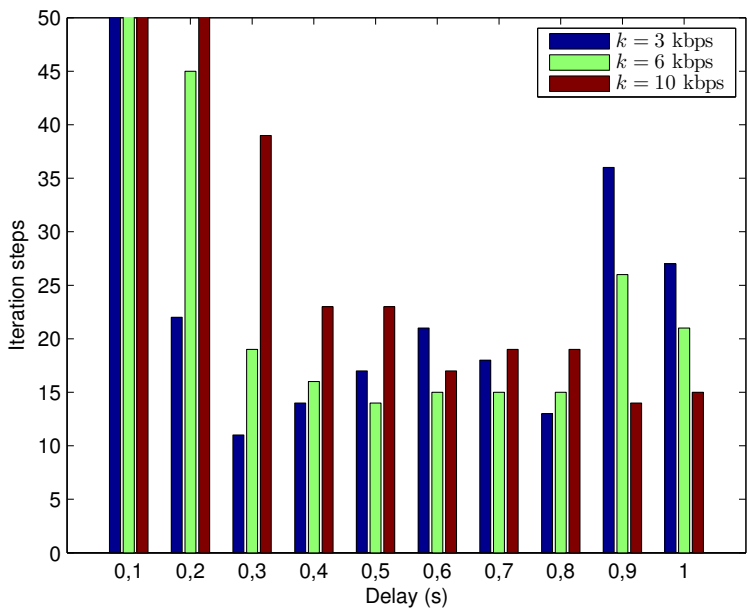

Fig. 7. Algorithm performance for different target delay bounds and data rates, where $\varepsilon=10^{-4}$.

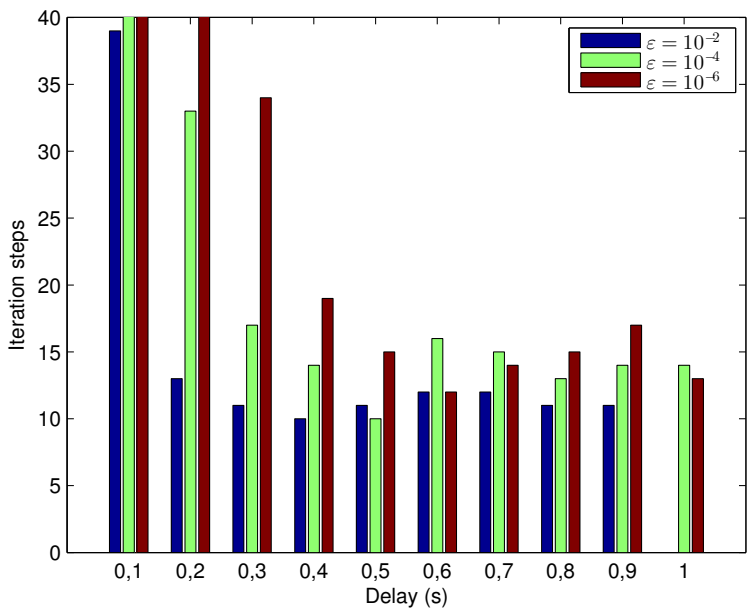

Fig. 8. Iteration steps for different target delay bounds and violation probabilities, where $k=5$ kbps.

value $f_{\text {stop }}$ and varies for different settings. For higher data rates, e.g. $k=10 \mathrm{kbps}$, the number of iterations decreases as the delay constraint is relaxed.

In Fig. 8 we present the algorithm performance for a traffic flow with data rate $k=5 \mathrm{kbps}$ and various QoS parameters $w$ and $\varepsilon$. We notice that for smaller delays and violation probabilities, the number of iterations exceeds 40 . As the target delay is relaxed, the algorithm needs less steps to reach the near-optimal SNR. Notice further, that for a fixed delay almost always the number of iterations increases as the violation probabilities become stricter. Moreover, as the QoS parameters become less strict, they can be fulfilled with $\bar{\gamma}=\bar{\gamma}_{\text {init }}$ and the number of iterations equals 0 . This is the case when $w=1 \mathrm{~s}$ and $\varepsilon=10^{-2}$.

Finally, in Fig. 9 we show the relationship between the number of iterations and the violation probability proximity factor $\Delta_{\varepsilon}$, defined in Eq. (15). The target violation probability is $10^{-5}$. As expected, the closer we want to come to the target $\varepsilon$, the more iterations are needed. Of course, the number of iterations is bigger in case of stricter delay constraints.

What we have further noticed throughout the numerical evaluations is that, the value $f_{\text {last }}$ is very often lower than 


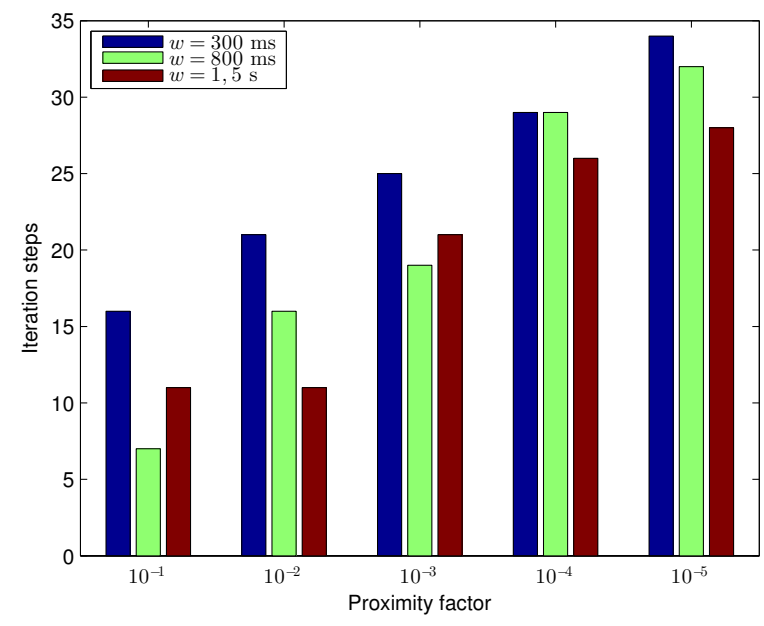

Fig. 9. Number of iterations vs. the violation probability proximity factor $\Delta_{\varepsilon}$ for different target delay bounds. The violation probability is $\varepsilon=10^{-5}$. The trend is obvious: The closer we want to get to $\varepsilon$, i.e. the bigger algorithm precision we want to achieve, the more iterations are needed.

$2^{-4}$, which results in an approximate distance to the actual minimal average SNR of $0.01 \mathrm{~dB}$ and smaller. This means that the optimum $\bar{\gamma}^{*}$ determined by the algorithm is very close to the optimal SNR $\bar{\gamma}_{\text {opt }}$. Notice further, that the number of iterations strongly depends on the initial value $\bar{\gamma}_{\text {init }}$, which in turn, in our case, depends on the data rate of the control application.

\section{CONCLUSION}

In this work we present an algorithm which determines a near-optimal average SNR, and therefore minimizes the transmit power of a sending node in a wireless industrial network. The traffic of interest is a QoS-constrained data flow, with a delay bound and its violation probability as QoS parameters, while the underlying system model is described with the WirelessHART standard. The algorithm is based on the definition for the delay bound function, defined with the $(\min , \times)$ network calculus in the SNR domain. It exploits the convexity and the monotonicity of this function and determines the near-optimal SNR by doing binary search along two dimensions. We have evaluated the algorithm performance for various settings and broad range of QoS requirements. The numerical results show that a near-optimal solution is found within a few iterations. We have further witnessed a tradeoff between the SNR and the target QoS system performance, which in turn reflects the number of needed iteration rounds of the proposed algorithm. In addition, we have identified several parameters which can be set in advance in order to influence the algorithm performance.

It is important to note that the algorithm can run on field devices in a WirelessHART network, as no high computational performance is necessary. In order to additionally accelerate the execution time, the computation of the incomplete gamma function can be implemented by using look-up tables. Hence, the nodes are going to be able to minimize their transmit power while fulfilling given statistical delay constraints and violation probabilities. This will result into increased energy efficiency and at the same time reduce potential interference. As a further step we plan to extend these evaluations to a multi-hop path and consider end-to-end performance constraints.

\section{ACKNOWLEDGMENT}

This work was partially funded by the programme for female doctoral candidates from the Fraunhofer Society. We further thank Barbara Staehle and Adriaan Schmidt for the helpful discussions and advices while working on the paper.

\section{REFERENCES}

[1] V. Gungor and G. Hancke, "Industrial Wireless Sensor Networks: Challenges, Design Principles, and Technical Approaches," Industrial Electronics, IEEE Transactions on, vol. 56, no. 10, pp. 4258-4265, Oct 2009.

[2] K. S. J. Pister and L. Doherty, "TSMP: Time Synchronized Mesh Protocol," in IASTED International Symposium on Distributed Sensor Networks. Acta Press, 2008, pp. 391-398.

[3] H. C. Foundation. (2013) Wirelesshart $\AA$ technology. [Online]. Available: http://www.hartcomm.org/

[4] ISA100 Commitee. (2014) ISA100, wireless systems for automation. [Online]. Available: www.isa.org/isa100

[5] H. Al-Zubaidy, J. Liebeherr, and A. Burchard, "A (min, $\times$ ) Network Calculus for Multi-Hop Fading Channels," in INFOCOM. IEEE, 2013, pp. 1833-1841.

[6] M. Gursoy, D. Qiao, and S. Velipasalar, "Analysis of Energy Efficiency in Fading Channels under QoS Constraints," Wireless Communications, IEEE Transactions on, vol. 8, no. 8, pp. 4252-4263, August 2009.

[7] D. Julian, M. Chiang, D. O'Neill, and S. Boyd, "QoS and Fairness Constrained Convex Optimization of Resource Allocation for Wireless Cellular and Ad Hoc Networks," in Proc. INFOCOM 2002., vol. 2, June 2002, pp. 477-486.

[8] M. Zafer and E. Modiano, "Delay-Constrained Energy Efficient Data Transmission over a Wireless Fading Channel," in Information Theory and Applications Workshop, 2007, Jan 2007, pp. 289-298.

[9] A. Fu, E. Modiano, and J. Tsitsiklis, "Optimal Energy Allocation for Delay-Constrained Data Transmission Over a Time-Varying Channel," in Proc. INFOCOM 2003, vol. 2, March 2003, pp. 1095-1105.

[10] M. Zafer and E. Modiano, "A Calculus Approach to Minimum Energy Transmission Policies With Quality of Service Guarantees," in Proc. INFOCOM 2005., vol. 1, March 2005, pp. 548-559.

[11] J. Lee and N. Jindal, "Energy-Efficient Scheduling of Delay Constrained Traffic Over Fading Channels," Wireless Communications, IEEE Transactions on, vol. 8, no. 4, pp. 1866-1875, April 2009.

[12] R. Berry and R. Gallager, "Communication Over Fading Channels With Delay Constraints," Information Theory, IEEE Transactions on, vol. 48, no. 5, pp. 1135-1149, May 2002.

[13] HART Communication Foundation. (2013) WirelessHART-How it works. [Online]. Available: http://de.hartcomm.org/protocol/wihart/ wireless_how_it_works.html

[14] Ieee standardization wpan task group. [Online]. Available: http: //www.ieee802.org/15/

[15] A. Goldsmith, Wireless Communications. Cambridge University Press, 2005.

[16] D. Tse, Fundamentals of Wireless Communications. Cambridge University Press, 2005.

[17] ZVEI - German Electrical and Electronic Manufacturers' Association. (2009) Coexistence of Wireless Systems in Automation Technology. [Online]. Available: http://www.zvei.org/Publikationen/ZVEI\%20Coexistence\%20of\% 20Wireless\%20Systems\%20in\%20Automation\%20Technology.pdf

[18] Y. Jiang and Y. Liu, Stochastic Network Calculus. Springer, 2008.

[19] B. Davies, Integral Transforms and Their Applications. SpringerVerlag, 1978. 